\title{
Leap-frog transport of magnetically driven anisotropic colloidal rotors
}

Cite as: J. Chem. Phys. 150, 164901 (2019); https://doi.org/10.1063/1.5086280

Submitted: 19 December 2018 . Accepted: 29 March 2019 . Published Online: 23 April 2019

Helena Massana-Cid, Eloy Navarro-Argemí, Demian Levis (D), Ignacio Pagonabarraga (D), and Pietro Tierno (D)

\section{COLLECTIONS}

Paper published as part of the special topic on Chemical Physics of Active Matter

Note: This article is part of the Special Topic "Chemical Physics of Active Matter" in J. Chem. Phys.
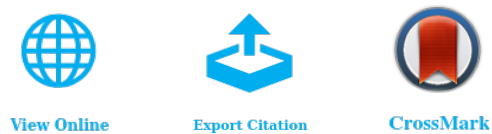

\section{ARTICLES YOU MAY BE INTERESTED IN}

Which interactions dominate in active colloids?

The Journal of Chemical Physics 150, 061102 (2019); https://doi.org/10.1063/1.5082284

Interparticle torques suppress motility-induced phase separation for rodlike particles The Journal of Chemical Physics 150, 164501 (2019); https://doi.org/10.1063/1.5086733

Statistical mechanics of transport processes in active fluids. II. Equations of hydrodynamics for active Brownian particles

The Journal of Chemical Physics 150, 164111 (2019); https://doi.org/10.1063/1.5054912

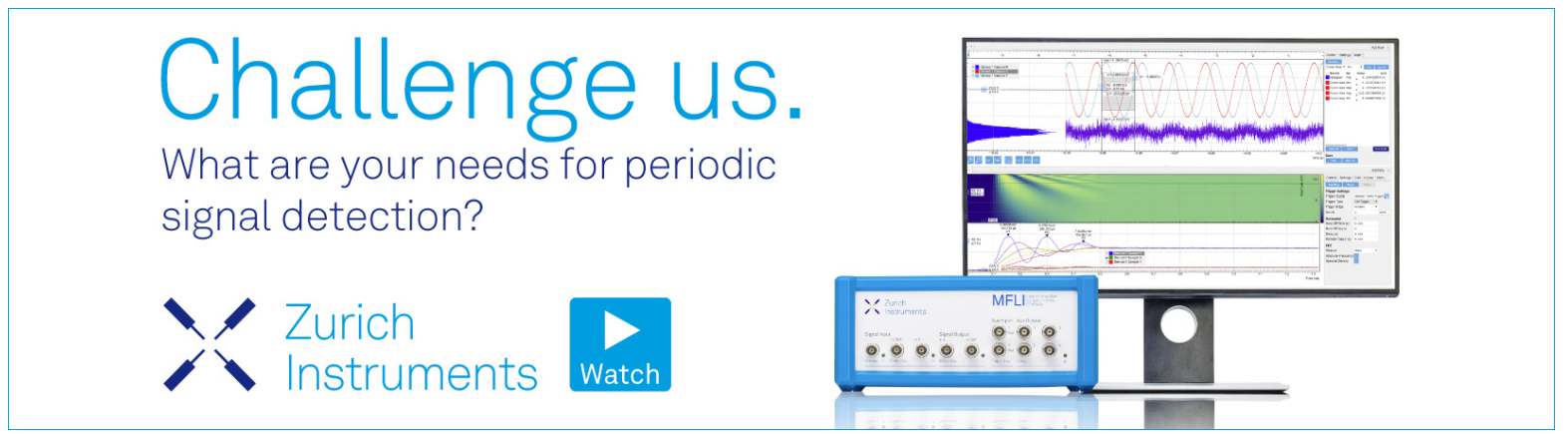




\title{
Leap-frog transport of magnetically driven anisotropic colloidal rotors
}

\author{
Cite as: J. Chem. Phys. 150, 164901 (2019); doi: 10.1063/1.5086280 \\ Submitted: 19 December 2018 - Accepted: 29 March 2019 • \\ Published Online: 22 April 2019
}

Helena Massana-Cid, ${ }^{1}$ Eloy Navarro-Argemí, ${ }^{1,2}$ Demian Levis, ${ }^{1,2,3}$ (D) Ignacio Pagonabarraga, and Pietro Tierno ${ }^{1,2,4, a)}$ (D)

\begin{abstract}
AFFILIATIONS
${ }^{1}$ Departament de Física de la Matèria Condensada, Universitat de Barcelona, 08028 Barcelona, Spain

${ }^{2}$ Universitat de Barcelona Institute of Complex Systems (UBICS), Universitat de Barcelona, 08028 Barcelona, Spain

${ }^{3}$ CECAM, Centre Européen de Calcul Atomique et Moléculaire, École Polytechnique Fédérale de Lasuanne, Batochime, Avenue Forel 2, 1015 Lausanne, Switzerland

${ }^{4}$ Institut de Nanociència i Nanotecnologia, IN² UB, Universitat de Barcelona, 08028 Barcelona, Spain
\end{abstract}

Note: This article is part of the Special Topic "Chemical Physics of Active Matter" in J. Chem. Phys.

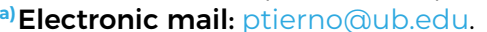

\begin{abstract}
In this article, we combine experiments and theory to investigate the transport properties of anisotropic hematite colloidal rotors that dynamically assemble into translating clusters upon application of a rotating magnetic field. The applied field exerts a torque to the particles forcing rotation close to a surface and thus a net translational motion at a frequency tunable speed. When approaching, pairs of particles are observed to assemble into stable three-dimensional clusters that perform a periodic leap-frog type dynamics and propel at a faster speed. We analyze the cluster formation and its lifetime and investigate the role of particle shape in the propulsion speed and stability. We show that the dynamics of the system results from a delicate balance between magnetic dipolar interactions and hydrodynamics, and we introduce a theoretical model that qualitatively explains the observed phenomena.
\end{abstract}

Published under license by AIP Publishing. https://doi.org/10.1063/1.5086280

\section{INTRODUCTION}

Recent years have witnessed a surge of activities in realizing artificial microscopic prototypes that could be remotely guided toward a defined target in a fluid medium. ${ }^{1-3}$ The driving interest arises from both fundamental and technological needs. The physics of artificial microswimmers navigating fluids fascinates scientists due its direct connection with biological systems, such as bacteria, that operate at the low Reynolds number. From the point of view of applications, artificial prototypes demonstrated the capabilities of performing useful tasks in microfluidic chips, ${ }^{4-6}$ in biomedicine, or to transport microscopic cargoes. ${ }^{9-15}$ Thus, the community has been mainly focused on designing individual microswimmers and understanding their principle of propulsion or on analyzing their collective dynamics in high density suspensions. ${ }^{16-23,37,38}$ However, investigating the interactions and dynamics between few micropropellers represents a first step toward understanding the complex phenomena arising from a collection of them. In particular, the use of colloidal particles as model microswimmers has the advantage that it avoids the intrinsic complexity of biological systems, providing tunable interactions and experimentally accessible length scales.

In this context, we recently realized controllable colloidal rotors that are composed of anisotropic hematite particles and are driven in water by an external rotating magnetic field. ${ }^{24}$ The particles rotate close to a surface, and the hydrodynamic interaction with the plane rectifies the rotational motion into a net drift velocity. ${ }^{4} \mathrm{We}$ find that when two rotors approach each other, they couple forming a dynamic bound state by adjusting their translational speed. The relative orientation of the particles within these states depends on the field parameters: the hematite ellipsoids align side-to-side or tip-to-tip, i.e., with the relative position parallel or perpendicular, respectively, to their long axis. However, in all the observed cases, the bound states remain confined in two dimensions with a 
relatively long interparticle distance. Here, by further exploring the space parameter, we discover a new type of cooperative dynamics, where a pair of propellers combines into a rolling three-dimensional cluster that displays a leap-frog type motion while propelling faster than individual rotors. We observe that this bound state can be formed by two or more rotors, and we characterize its stability and dynamics by varying the different field parameters. We complement these experiments with a theoretical model that considers the hydrodynamic interactions between the particles and the bounding plane.

\section{EXPERIMENTAL PART}

We realize micropropellers by using anisotropic hematite particles that are synthesized via the "sol-gel" technique. ${ }^{25}$ The particles present a long and a short axis equal to $\alpha=2.5 \mu \mathrm{m}$ and $\beta=1.4 \mu \mathrm{m}$, respectively, and a permanent magnetic moment $m$ perpendicular to their long axis, as shown in the schematic of Fig. 1(a). In a previous studies, ${ }^{24,39}$ the magnitude of this moment was measured as $m \simeq 9 \times 10^{-16} \mathrm{Am}^{2}$. After synthesis, the particles are diluted in highly deionized water (Milli-Q system, Millipore) and are stabilized by adding sodium dodecyl sulfate (SDS), namely, $0.11 \mathrm{~g}$ of SDS for $80 \mathrm{ml}$ of water. This surfactant is used to avoid sticking of the particles due to van der Waals interactions. Before the experiments, the $\mathrm{pH}$ of the resulting solution is raised to 9.5 by adding tetramethylammonium hydroxide. The particles sediment on a glass surface, and the balance between gravity and steric interactions due to the SDS confines them on the plane.

The external magnetic field used to propel the particles is generated by using a triaxial magnetic coil system connected to a waveform generator (TGA1244, TTi) feeding a power amplifier (AMP-1800, AKIYAMA or BOP 20-10M, Kepco). A rotating field in a given plane is obtained by passing through two perpendicular coils two sinusoidal currents with $90^{\circ}$ phase shift. We then use optical microscopy (Eclipse Ni, Nikon) to record the particle motion with an area-scan camera (scA640-74f, Basler) working at 50 fps. The videos are then analyzed via particle tracking routines $^{26}$ to extract the position and the orientation of the hematite particles.

\section{DYNAMICS OF AN INDIVIDUAL ROTOR}

We start by describing the propulsion of a single rotor and later focus on the emergence of the three-dimensional leap-frog states. The particles are propelled by applying a rotating magnetic field circularly polarized in the $(\hat{x}, \hat{z})$ plane that is perpendicular to the glass substrate, as shown in Fig. 1(a). The applied field is given by $\boldsymbol{H}=H_{0}(\cos (\omega t) \hat{x}-\sin (\omega t) \hat{z})$, being $H_{0}$ the amplitude and $\omega$ the driving frequency. Figure 1 (b) shows two snapshots of a dilute suspension of rotors driven toward the right by the rotating field. Since the permanent moment of the particles is directed along the short axis, Fig. 1(a), during propulsion, the particles keep their long axis always perpendicular to the direction of motion, here given by $\hat{x}$. From particle tracking, we extract the position of each particle $i$, $\left(x_{i}(t), y_{i}(t)\right)$, and determine the mean speed of the individual rotors as $\left\langle v_{x}\right\rangle=\frac{1}{N} \sum_{i}^{N} \frac{\Delta x_{i}}{\Delta t}$, where the average $\langle\ldots\rangle$ is performed over $N$ different and independent particles. As shown in Fig. 1(c), the particle dynamics is characterized by two regimes of motion, separated by a critical frequency $\omega_{c}$. The synchronous transport occurs for $\omega<\omega_{c}$, where the magnetic moment of the particle follows the applied field, and thus, the particle rotational frequency $\Omega$ coincides with the driving one, $\Omega=\omega$. In this situation, assuming that the particles have an elevation from the surface equal to $\sim \beta / 2$, the net drift velocity is given by $v_{x} \sim \beta \omega / 2$. The dashed lines in Fig. 1(c) show the corresponding fit to the experimental data in this linear regime. For $\omega>\omega_{c}$, the motion becomes asynchronous, and the phase lag angle between the particle's moment and the field is not constant. This effect induces characteristic back-and-forth oscillations during each field cycle and a consequent reduction of the average particle rotational motion. Neglecting thermal fluctuations, the average speed of the particles can be calculated $\operatorname{as}^{27} v_{x} \sim \beta \Omega / 2$ with
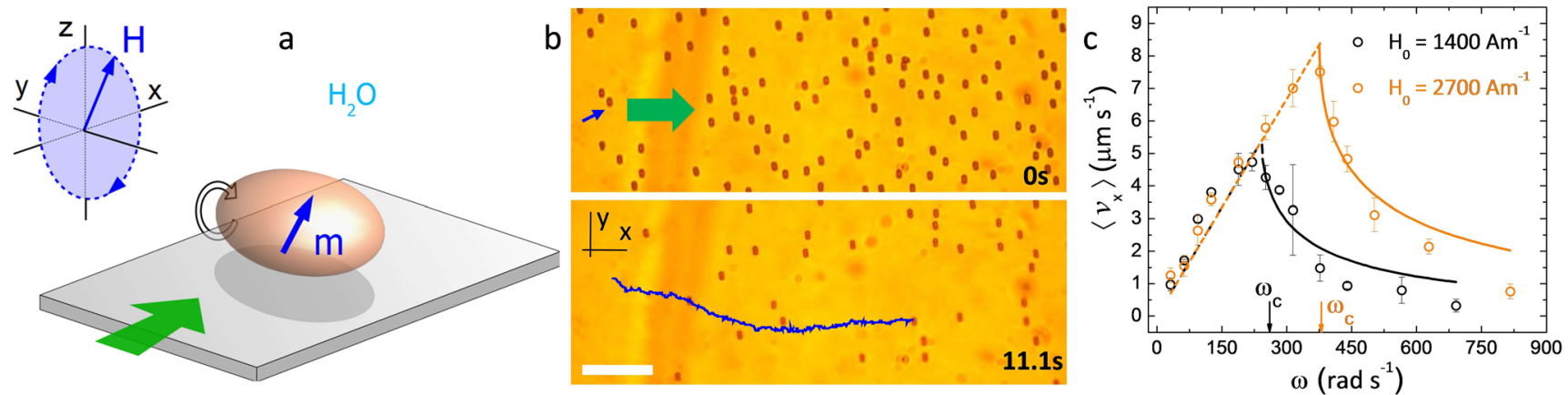

FIG. 1. (a) Schematic illustrating a ferromagnetic hematite ellipsoidal particle propelling in water due to the application of a rotating magnetic field $\boldsymbol{H}$. Green arrows indicate the direction of propulsion. (b) Sequence of images showing a dilute solution of hematite particles that propel toward the right due to a rotating field with amplitude $H_{0}=$ $2700 \mathrm{~A} \mathrm{~m}^{-1}$ and angular frequency $\omega=251.3 \mathrm{rad} \mathrm{s}^{-1}$. In the bottom image, the trajectory of one particle is superimposed and the scale bar is $20 \mu \mathrm{m}$. (c) Average speed $\left\langle v_{x}\right\rangle$ vs driving frequency $\omega$ for a single propeller when subjected to a rotating field with amplitudes $H_{0}=1400 \mathrm{~A} \mathrm{~m}^{-1}$ (black circles) and $H_{0}=2700 \mathrm{~A} \mathrm{~m}{ }^{-1}$ (orange circles). Dashed (continuous) lines are fit to the synchronous (asynchronous) regime, while $\omega_{c}$ is the critical frequency. Error bars are obtained from the statistical average of different experiments. 
$\Omega=\omega-\sqrt{\omega^{2}-\omega_{c}^{2}}$, as shown by the continuous lines in Fig. 1(c). From the fits to the experimental data, we determine the critical frequencies for the two field amplitudes $\omega_{c}=243 \pm 3 \mathrm{rad} \mathrm{s}^{-1}$ for $H_{0}=1400 \mathrm{~A} \mathrm{~m}^{-1}$ and $\omega_{c}=376 \pm 3 \mathrm{rad} \mathrm{s}^{-1}$ for $H_{0}=2700 \mathrm{~A} \mathrm{~m}^{-1}$, which, as previously observed, ${ }^{27}$ increase linearly with $H_{0}$. Thus, our hematite rotors display a tunable speed that increases linearly with the driving frequency up to $\omega_{c}$, while this threshold frequency could be further raised by tuning the field amplitude.

\section{LEAP-FROG DYNAMICS OF ROTORS}

At large density of rotors, propelling particles start to interact with each other. Very close hematite rotors may couple their motion forming bound states and altering their translational speed. As shown in the diagram in Fig. 2(a), we observe different situations depending on whether the motion occurs on the same plane or becomes three-dimensional (leap-frog state). For high driving frequencies, we observe that the rotating particles never come too close to generate the leap-frog state, but rather they bound into metastable states confined in the plane of motion $(\hat{x}, \hat{y})$. In this situation, the
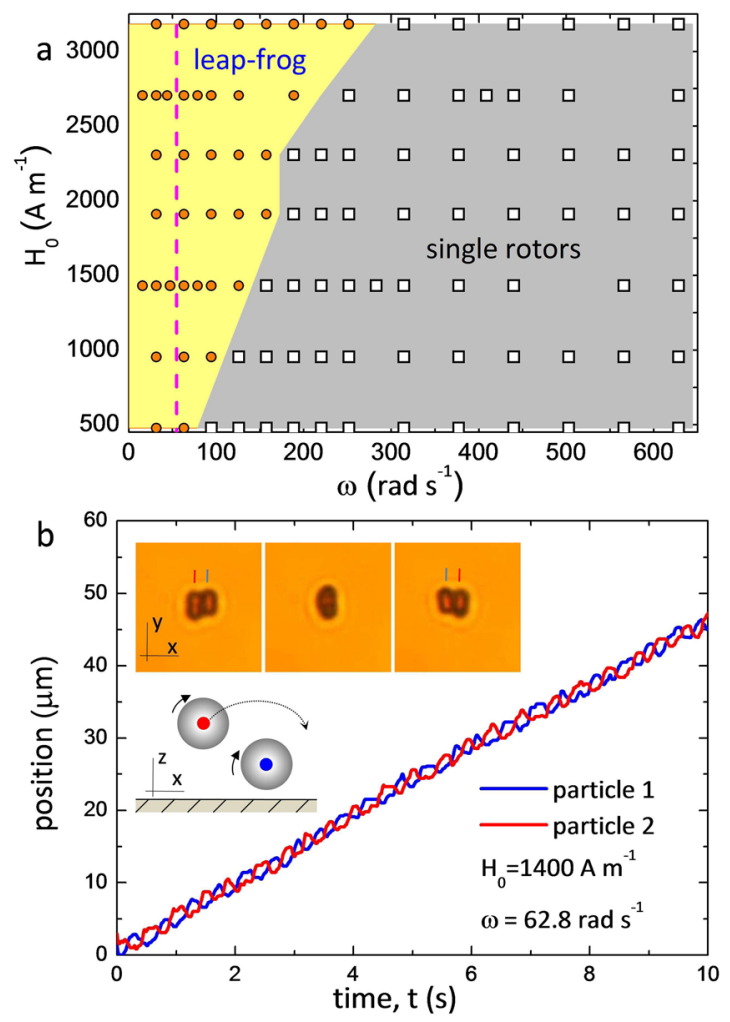

FIG. 2. (a) Diagram in the $\left(\omega ; H_{0}\right)$ plane showing the regime where the leap-frog dynamics is observed (yellow region) and where the movement of the hematite particle is limited to a $2 \mathrm{D}$ plane (gray region). The magenta dashed line corresponds to $\omega=54.8 \mathrm{rad} \mathrm{s}^{-1}$. (b) Position vs time of two particles performing the leap-frog dynamics. Insets at the top show experimental images of the hematite particles exchanging their positions during propulsion and the microscope image visualizes the $(\hat{x}, \hat{y})$ plane at a fixed height. The inset at the bottom shows the corresponding schematic in the $(\hat{x}, \hat{z})$ plane. relative particle arrangement is the same to that reported in a previous study ${ }^{24}$ and thus here will not be described. By contrast, at low frequency, we find that the pair of approaching particles can form three-dimensional leap-frog states, where the hematite ellipsoids roll one on top of each other, periodically exchanging their positions and always keeping their long axis perpendicular to the propulsion direction [Fig. 2(b)].

The resulting dynamics is mainly dictated by the balance between the magnetic dipolar and hydrodynamic interactions. The first type of interaction favors that the particles arrange side by side with their long axis parallel to each other. In this configuration, the permanent moments tend to be aligned along a common direction in order to minimize the magnetic energy of the couple, $U_{m}$. If we consider two hematite rotors with moments $\boldsymbol{m}_{i}$, $\boldsymbol{m}_{j}$ and at a relative distance $\boldsymbol{r}_{i j}=\left|\boldsymbol{r}_{i}-\boldsymbol{r}_{j}\right|$, the dipolar interaction between the couple is given by $U_{m}=\frac{\mu_{w}}{4 \pi}\left(\frac{\boldsymbol{m}_{i} \boldsymbol{m}_{j}}{r_{i j}^{3}}-\frac{3\left(\boldsymbol{m}_{i} \cdot \boldsymbol{r}_{i j}\right)\left(\boldsymbol{m}_{j} \cdot \boldsymbol{r}_{i j}\right)}{r_{i j}^{5}}\right)$. Here, $\mu_{w} \sim \mu_{0}=4 \pi \cdot 10^{-7} \mathrm{H} \mathrm{m}$ is the magnetic susceptibility of the medium (water) and $\boldsymbol{r}_{i}$ is the position of particle $i$. Thus, the dipolar potential is maximally attractive (repulsive) for particles with magnetic moments parallel (perpendicular) to $\boldsymbol{r}_{i j}$. Since the moment of the particles follows the field that rotates in the $(\hat{x}, \hat{z})$ plane, the leapfrog motion minimizes the potential $U_{m}$. This follows from the fact that the distance between $\boldsymbol{m}_{i}$ and $\boldsymbol{m}_{j}$ is shorter (longer) when $\boldsymbol{m}_{i, j} \| \boldsymbol{r}_{i j}\left(\boldsymbol{m}_{i, j} \perp \boldsymbol{r}_{i j}\right)$ during one field cycle. The time average potential between two dipoles performing the leap-frog motion is indeed attractive in the plane $(x, z)$ and given by $\bar{U}_{m}=-\mu_{0} m^{2} /\left(8 \pi(x+z)^{3}\right)$. In the opposite case, when the particles are aligned tip to tip on the same plane, the moments are always perpendicular to their separation distance, and the potential becomes repulsive $\bar{U}_{m}=\mu_{0} m^{2} /\left(4 \pi y^{3}\right)$.

This configuration, however, could be perturbed by the hydrodynamic interactions (HIs), which arise from the particle spinning and the generated vortical flow field. The order of magnitude of the two competing interactions can be estimated, in first approximation, by considering the range of forces that act over the pair. The magnetic dipolar forces $F_{m}$ between two equal dipolar particles of radius $a$ and moment $m$ and located at distance $r=2 a$ is given by $F_{m}=3 \mu_{0} \mathrm{~m}^{2} /\left(64 \pi a^{4}\right)$, while the viscous force generated by a single hematite particle is $F_{h}=3 \pi \eta \Omega h^{2} / 4$, being $\eta=10^{-3} \mathrm{~Pa}$ s the solvent viscosity and $h \sim a$ the particle elevation from the surface. Hence, for $a \sim \beta / 2=0.7 \mu \mathrm{m}$, we find that when both forces balance, $F_{m}=F_{h}$, the angular frequency becomes $\Omega=\mu_{0} m^{2} /\left(16 \pi^{2} \eta a^{6}\right)$ $=54.8 \mathrm{rad} \mathrm{s}^{-1}$, which corresponds to the dashed line in Fig. 2(a). Thus, at low driving frequencies, magnetic interactions dominate over HIs and help keeping the leap-frog stable, as shown in the diagram in Fig. 2(a). However, our simple argument does not explain how the transition region depends on the field amplitude. Indeed, at large values of $H_{0}$, the presence of an induced dipole moment $m_{\text {ind }}$ $\sim H_{0}$ within the particle may lead to strong dipolar interactions and further favor the leap-frog state. While magnetization measurements of similar particles show a negligible value of $m_{i n d},{ }^{27}$ its contribution to $F_{m}$ may become important for large amplitudes, as it grows as $\sim H_{0}^{2}$.

In Fig. 3(a), we measure the average speed $\left\langle v_{x}\right\rangle$ of a cluster of hematite particles performing the leap-frog and compare its values to the speed of individual rotors in the synchronous regime. The cluster displays a faster translational motion until it reaches the 


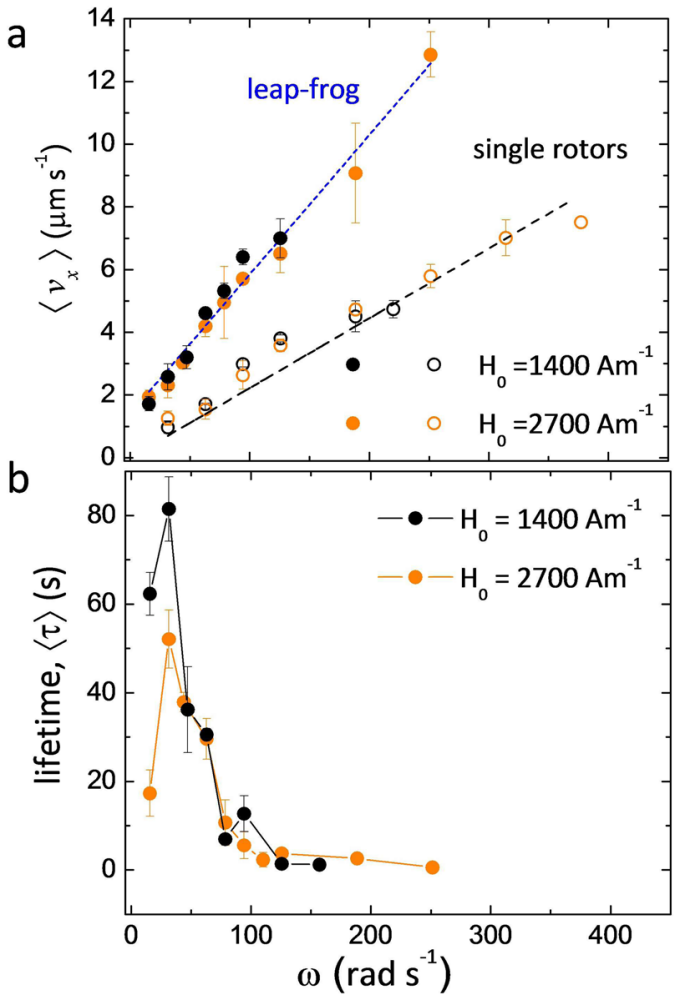

FIG. 3. (a) Average propulsion speed $\left\langle v_{x}\right\rangle$ vs angular driving frequency $\omega$ for single rotors (open symbols) and for a leap-frog state (filled symbols). The straight lines are linear fits that serve as guides to the eyes. (b) Average lifetime $\langle\tau\rangle$ vs $\omega$ for a bound state performing the leap-frog dynamics for two field amplitudes. In both graphs, the error bars are obtained from the statistical average of different experiments.

critical frequency $\omega_{c}$, where the two particles enter the asynchronous regime reducing their rotational motion. In this case, the couple easily breaks due to the difference in speeds between the two hematite rotors. Furthermore, we report in Fig. 3(b) the average lifetime $\langle\tau\rangle$ of the observed leap-frog states for two different field amplitudes. The presence of thermal noise or disorder in the experimental system may produce the disassembly of the pair during propulsion, even at frequencies well inside the synchronous regime. The disorder may be due to small polydispersity in the particle shape, in their magnetic moments, or to the presence of surface asperities. These sources of noise can induce variations of the hydrodynamic flow that easily destabilize the propelling cluster. In general, we find that stable clusters with longer $\langle\tau\rangle$ are observed at low frequencies when magnetic dipolar interactions completely overcome the hydrodynamic ones.

\section{THEORETICAL MODEL}

We describe the HIs between a pair of particles performing the leap-frog state by considering the direct interactions and the presence of the bounding plane. The surface is replaced by an hydrodynamic singularity located below the interface at the same distance to it as the colloidal particle. The latter image is represented by a particle rotating in the opposite sense that the actuated colloid, and contributing to the flow field with additional stresslet and source doublets. ${ }^{28}$ We neglect the magnetic dipolar interactions between the pair and assume that the imposed rotating field simply prescribes the angular velocity of the colloid $\Omega$. Thus, in the synchronous regime, the colloids of radius $a$ rotate at $\Omega=\omega$. In the far field, the flow generated by one of them is given by

$$
\begin{aligned}
\frac{u_{i}}{a^{3}}= & \frac{\epsilon_{i j k} \Omega_{j} r_{k}}{r^{3}}-\frac{\epsilon_{i j k} \Omega_{j} R_{k}}{R^{3}} \\
& +2 h \epsilon_{k j z} \Omega_{j}\left(\frac{\delta_{i k}}{R^{3}}-\frac{3 R_{i} R_{k}}{R^{5}}\right)+6 \epsilon_{k j z} \frac{\Omega_{j} R_{i} R_{k} R_{z}}{R^{5}},
\end{aligned}
$$

where $r$ is the position vector from the center of the particle, $R$ is the position of its image, and the subscripts $(i, j, k, z)$ refer to vector components. From Eq. (1), we can derive analytic expressions for the speeds $\boldsymbol{v}_{i}$ of the two spheres $i=1,2$ rotating with angular speed $\Omega_{i}$ and at elevation $h_{i}$; see the Appendix for the full expressions. To explore the role of anisotropy on the leap-frog state, we perform numerical simulation of single spheres rotating at a common angular velocity and of colloidal dumbbells. We model a dumbbell as a pair of spherical particles linked by a harmonic potential with a stiff spring constant that allows the particle rotation but not separation between the pair. Thus, the dumbbells are made of a pair spheres $(i, i+1)$ bounded by a harmonic potential

$$
V\left(r_{i, i+1}=r\right)=\frac{k}{2}\left(r-r_{0}\right)^{2},
$$

where $r_{0}$ defines the distance between the two composing spheres at rest and $k$ is a spring constant. Thus, each sphere evolves according to an overdamped dynamics

$$
\gamma \dot{\boldsymbol{r}}_{i}=\gamma \boldsymbol{v}_{i}+\boldsymbol{f}_{i},
$$

where $\gamma$ is the viscous friction and $\boldsymbol{f}_{i}=-\nabla_{i} V\left(\boldsymbol{r}_{i, i+1}\right)$ is the spring force. We choose a relatively very stiff spring $\left(k=10^{3}\right)$ that guarantees the dumbbell stability $(k \gg \Omega \gamma a)$ avoiding particle separation and set $r_{0}=a$.

Figures 4(a)-4(d) show the results of our numerical simulations based on the hydrodynamic model. We start by first calculating the flow profile generated by a pair of spherical rotors that moves toward right along the $\hat{x}$ direction side by side [Fig. 4(a)] and one on top of the other [Fig. 4(b)]. In the first case, the effective flow field induced by the pair creates an elongated dipole with a source corresponding to the front particle and a sink on the back one. When one particle is above the other, the two flow patterns superimpose, creating two lateral swirls with opposite rotational motion. Thus, tracer particles could be easily transported by the propelling pair, being attracted to it when at the back of the pair and pushed when in front of it.

Furthermore, we explore the role of shape anisotropy on the propulsion speed and on the formation of the leap-frog states. As shown in Fig. 4(c), we find that single spheres and dumbbells present almost the same speed that linearly increases with $\omega$. The main difference arises when the pair of spheres or dumbbells performs the leap-frog as we observe a much faster propulsion speed for the latter. However, we find that the normalized velocity $\left\langle v_{x}\right\rangle$ overshoots the experimental data by almost a factor of two. This 

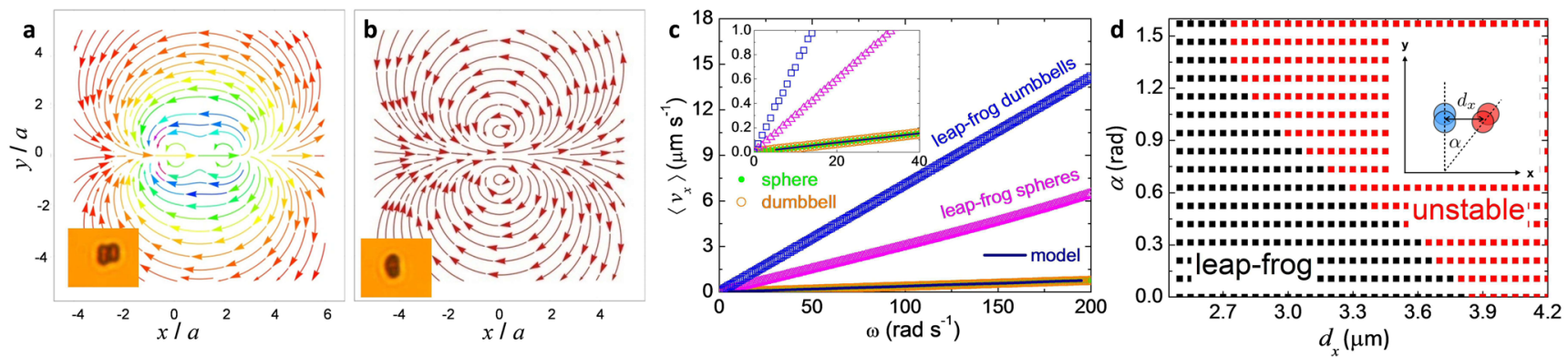

FIG. 4. [(a) and (b)] Plot of the streamlines of the fluid flow velocity $\mathbf{u}$ generated by a pair of spherical rotors performing the leap-frog motion before one particle jumps on top of the other (a) and when the particle sits on top of the other. The $(x, y)$ coordinates are normalized with respect to the particle radius a. The velocity field was calculated in (a) considering both particles at the same elevation $\left(z_{1}=z_{2}=2 a\right)$ and in (b) with different elevations $\left(z_{1}=a ; z_{2}=3 a\right)$ being a the particle radius. The motion of the particles occurs toward the right. (c) Average speed $\left\langle v_{x}\right\rangle$ vs frequency for a single sphere (green filled points) and dumbbell (orange circles) and for a pair of spherical rotors (magenta triangles) and dumbbells (blue squares) performing leap-frog motion. The continuous line is obtained from Eq. (1) in the main text. The inset illustrates enlargement of the main graph. (d) Diagram illustrating the regions where the leap -frog is stable (black squares) and not (red squares) by varying the initial distance $d_{x}$ and orientation angle $\alpha$ between the two dumbbells. The schematic inset shows $\alpha$ and $d_{x}$.

discrepancy could result from the different simplifications used in our model. Specifically, the theoretical model disregards both gravity and magnetic interactions and neglects the homogeneous surface of the particles by considering them as dumbbells. Nevertheless, we find that the leap-frog states could be formed by both spherical and anisotropic rotors, which does not limit our mechanism to magnetic particles with anisotropic shape but also to other types of colloidal systems. ${ }^{29}$

We also observe that the leap-frog state is much more stable for spherical rotors than for pairs of dumbbells. In the first case, according to the hydrodynamic model, we find that there is no preferred distance between two particles to generate the leapfrog motion. Thus, identical spherical particles will always be coupled, unless thermal dissipation or other types of noise break the bound state. This contrasts with our experimental observation of a finite lifetime [Fig. 3(b)] and can be explained by considering the anisotropic shape of the particles and the presence of a relative orientational motion. In Fig. 4(d), we show the stability of a pair of dumbbells performing leap-frog by varying their initial distance $d_{x}$ and orientation angle $\alpha$. We define the stable leap-frog when it lasts for all the simulation time ( $1000 \Omega^{-1}$ with $\Omega=200 \mathrm{rad} \mathrm{s}^{-1}$ ) while unstable when it breaks, or does not form at all, as observed for larger distance or angle between the dumbbells. Leap-frog motion might be broken by thermal fluctuations, or other sources noise such as particle inhomogeneities, tumbling induced by particle collision, and substrate roughness. These factors may easily change the distance and relative orientation or even change the particle speed as the cluster of rotors could develop a self-propelling velocity that depends on their elevation (the closer they are, the faster they move).

\section{TRANSPORT OF SEVERAL PARTICLES}

The leap-frog state is reminiscent of the walking-like dynamics observed both on dimers ${ }^{30,31}$ and clusters ${ }^{32,33}$ assembled from paramagnetic colloids. However, in contrast to the mentioned studies,
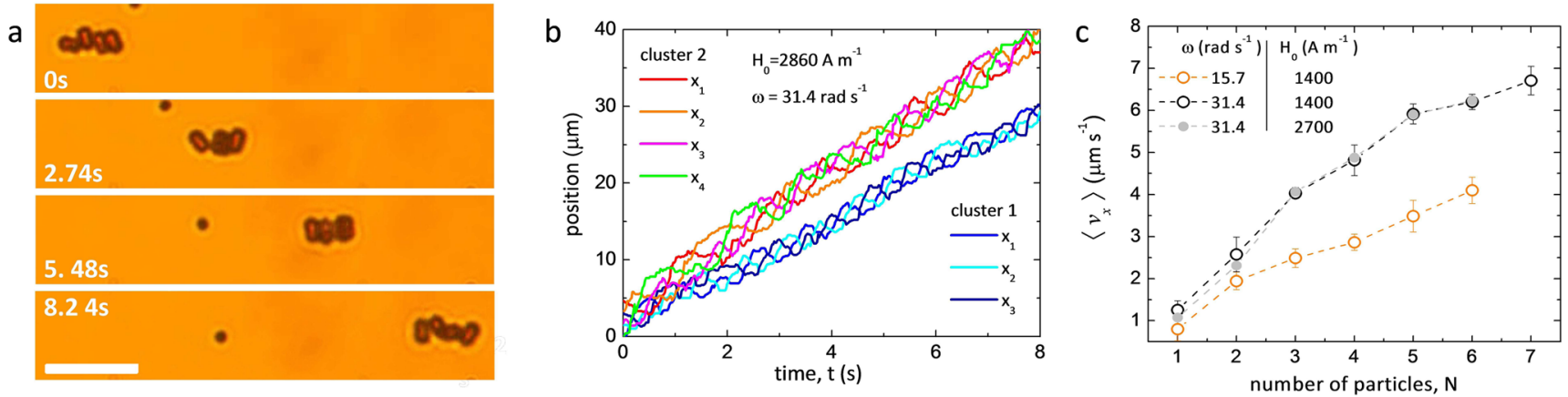

FIG. 5. (a) Sequence of optical microscopy images illustrating the transport of a cluster formed by 4 hematite rotors that move toward the right. The scale bar at the bottom is $10 \mu \mathrm{m}$. (b) Position ( $\hat{x}$ ) vs time of two clusters composed by 3 (cluster 1 ) and 4 [cluster 2, image (a)] particles propelling toward the right. (c) Average speed $\left\langle v_{x}\right\rangle$ vs number of particles $N$ of a propelling cluster for two different driving frequencies and field amplitudes. The error bars are obtained from the statistical average of different experiments. 
here, we find that increasing the number of particles, the leapfrog extends along the $\hat{x}$ direction, thus parallel to the plate, rather than forming tumbling columns perpendicular to it. This situation is illustrated in Fig. 5(a), where a cluster composed by 4 hematite rotors translates toward the right at an average speed of $\left\langle v_{x}\right\rangle=5$ $\mu \mathrm{m} \mathrm{s}^{-1}$. During propulsion, particles forming the chain are expelled from it to the top, roll on top of the chain, and re-attach at the front of it creating the pair of propelling leap-frog states. This process can be appreciated by tracking the position of the individual particles within smaller clusters, as shown in Fig. 5(b) for two particular cases. If we consider "cluster 2" composed by 4 particles, the positions of each particle periodically alternate with short and long periods where the slope, and thus the velocity, changes or it remains constant, respectively. This indicates that the particles approach the edges of the cluster and slow down, while when they slide above the other 3 particles, they display a constant speed. This process becomes more evident for longer clusters containing more than 6 particles. However, we find that these structures are more difficult to track due to the continuous particle overlapping that impedes the identification of the single elements within a cluster. The average speed along the propulsion direction for different number of particles $N$ is shown in Fig. 5(b). Here, we measure the average speed of the center of symmetry of the cluster that approximately coincides with the center of velocity. We find that larger clusters propel faster until reaching a saturation value for $N \sim 7$. The general trend is similar to that reported for paramagnetic colloids assembled into two-dimensional carpets, where the speed rapidly increases at the beginning and then it saturates at a large value of $N$. We have explained this behavior in terms of the hydrodynamic coupling among the colloids. Generically, beyond a characteristic length of the chain, the hematite rotors composing the colloidal structure start to be far away, and the relevance of flow additivity vanishes.

\section{CONCLUSIONS}

We have investigated the transport properties of anisotropic hematite colloidal rotors that are driven in a viscous fluid via an external rotating magnetic field. We find that close propelling rotors assemble into rotating clusters performing a leap-frog type dynamics. The clusters display an enhancement of their average speed which arises from the hydrodynamic coupling between the pair. We explain the propulsion using the far field approximation by extending Blake's tensor formalism to our system. ${ }^{28}$ We further investigate the role of shape on the dynamics of the pair by comparing numerical simulations of single spheres with dumbbells. We find that while both types of rotors display the leap-frog state, the orientational degrees of freedom of the dumbbells make such a state more unstable.

Our colloidal rollers require a confined geometry to propel and thus could be implemented in microfluidic environments in order to trap, control, and transport micro-objects in a fluid medium. Indeed, previous experimental studies based on paramagnetic colloids, ${ }^{34}$ hematite-polystyrene composite, ${ }^{35}$ and ellipsoidal particles ${ }^{36}$ showed promises in this direction. In our particular case, one could use the pair of rotors to transport a biological cargo entrapped in their vortical flow and then release it by simply varying the frequency of the applied field that allows to tune the lifetime of the leap-frog state. From a more fundamental perspective, our work invites further investigations related to the role of hydrodynamic interactions in synchronization phenomena between emerging suspensions of actuated magnetic colloids.

\section{ACKNOWLEDGMENTS}

H.M.-C. and P.T. acknowledge support from the ERC Grant "DynaMO" (No. 335040). E.N.A. and I.P. acknowledge support from MINECO (Grant No. FIS2015-67837-P) and DURSI (Grant No. 2014SGR-922). P.T. acknowledges support from MINECO (Grant Nos. FIS2016-78507-C2-2-P and ERC2018-092827), DURSI (2017SGR1061), and Generalitat de Catalunya under program "Icrea Academia."

\section{APPENDIX: DETAILS ON THE THEORETICAL MODEL}

For two particles of radius $a_{i}$ located at positions $\left(x_{i}, y_{i}, h_{i}\right)$ with an angular velocity $\vec{\Omega}_{i}=\Omega_{i} \hat{y}$, the velocities at which they will displace are given by

$$
\left\{\begin{array}{l}
v_{x, 1}=\frac{\Omega_{1} a_{1}^{5}}{8 h_{1}^{4}}+\Omega_{2} a_{2}^{3}\left(6 h_{1} \frac{\left(x_{1}-x_{2}\right)^{2}}{\left(\Delta^{2}+\left(h_{1}+h_{2}\right)^{2}\right)^{5 / 2}}+\frac{h_{1}-h_{2}}{\left(\Delta^{2}+\left(h_{1}-h_{2}\right)^{2}\right)^{3 / 2}}+\frac{h_{2}-h_{1}}{\left(\Delta^{2}+\left(h_{1}+h_{2}\right)^{2}\right)^{3 / 2}}\right) \\
v_{x, 2}=\frac{\Omega_{2} a_{2}^{5}}{8 h_{2}^{4}}+\Omega_{1} a_{1}^{3}\left(6 h_{2} \frac{\left(x_{1}-x_{2}\right)^{2}}{\left(\Delta^{2}+\left(h_{1}+h_{2}\right)^{2}\right)^{5 / 2}}+\frac{h_{2}-h_{1}}{\left(\Delta^{2}+\left(h_{1}-h_{2}\right)^{2}\right)^{3 / 2}}+\frac{h_{1}-h_{2}}{\left(\Delta^{2}+\left(h_{1}+h_{2}\right)^{2}\right)^{3 / 2}}\right) \\
v_{y, 1}=6 h_{1} \Omega_{2} a_{2}^{3} \frac{\left(x_{2}-x_{1}\right)\left(y_{2}-y_{1}\right)}{\left(\Delta^{2}+\left(h_{1}+h_{2}\right)^{2}\right)^{5 / 2}}, \\
v_{y, 2}=6 h_{2} \Omega_{1} a_{1}^{3} \frac{\left(x_{2}-x_{1}\right)\left(y_{2}-y_{1}\right)}{\left(\Delta^{2}+\left(h_{1}+h_{2}\right)^{2}\right)^{5 / 2}}, \\
v_{z, 1}=\Omega_{2} a_{2}^{3}\left(\frac{x_{2}-x_{1}}{\left(\Delta^{2}+\left(h_{1}-h_{2}\right)^{2}\right)^{3 / 2}}+\frac{x_{1}-x_{2}}{\left(\Delta^{2}+\left(h_{1}+h_{2}\right)^{2}\right)^{3 / 2}}+6 h_{1} \frac{\left(x_{1}-x_{2}\right)\left(h_{1}+h_{2}\right)}{\left(\Delta^{2}+\left(h_{1}+h_{2}\right)^{2}\right)^{5 / 2}}\right) \\
v_{z, 2}=\Omega_{1} a_{1}^{3}\left(\frac{x_{1}-x_{2}}{\left(\Delta^{2}+\left(h_{1}-h_{2}\right)^{2}\right)^{3 / 2}}+\frac{x_{2}-x_{1}}{\left(\Delta^{2}+\left(h_{1}+h_{2}\right)^{2}\right)^{3 / 2}}+6 h_{2} \frac{\left(x_{2}-x_{1}\right)\left(h_{1}+h_{2}\right)}{\left(\Delta^{2}+\left(h_{1}+h_{2}\right)^{2}\right)^{5 / 2}}\right)
\end{array}\right.
$$


with $\Delta^{2} \equiv\left(x_{2}-x_{1}\right)^{2}+\left(y_{2}-y_{1}\right)^{2}$.

\section{REFERENCES}

${ }^{1}$ M. C. Marchetti, J. F. Joanny, S. Ramaswamy, T. B. Liverpool, J. Prost, M. Rao, and R. A. Simha, Rev. Mod. Phys. 85, 1143 (2013).

${ }^{2}$ C. Bechinger, R. D. Leonardo, H. Löwen, C. Reichhardt, G. Volpe, and G. Volpe, Rev. Mod. Phys. 88, 045006 (2016).

${ }^{3}$ K. Han, W. C. S. IV, and O. D. Velev, Adv. Funct. Mater. 28, 1705953 (2018).

${ }^{4}$ P. Tierno, R. Golestanian, I. Pagonabarraga, and F. Sagués, J. Phys. Chem. B 112, 16525 (2008)

${ }^{5}$ J. Burdick, R. Laocharoensuk, P. M. Wheat, J. D. Posner, and J. Wang, J. Am. Chem. Soc. 130, 8164 (2008)

${ }^{6}$ L. Baraban, D. Makarov, R. Streubel, I. Mönch, D. Grimm, S. Sanchez, and O. G. Schmidt, ACS Nano 6, 3383 (2012).

${ }^{7}$ S. Guo, Q. Pan, and M. B. Khamesee, Microsyst. Technol. 14, 307 (2008).

${ }^{8}$ B. J. Nelson, I. K. Kaliakatsos, and J. J. Abbott, Annu. Rev. Biomed. Eng. 12, 55 (2010).

${ }^{9}$ R. Dreyfus, J. Baudry, M. L. Roper, M. Fermigier, H. A. Stone, and J. Bibette, Nature 437, 862 (2005).

${ }^{10}$ A. Snezhko and I. S. Aranson, Nat. Mater. 10, 698 (2011).

${ }^{11}$ T. Petit, L. Zhang, K. E. Peyer, B. E. Kratochvil, and B. J. Nelson, Nano Lett. 12, 156 (2012).

${ }^{12}$ J. Palacci, S. Sacanna, A. P. Steinberg, D. J. Pine, and P. M. Chaikin, Science 339, 936 (2013).

${ }^{13}$ F. Martinez-Pedrero and P. Tierno, Phys. Rev. Appl. 3, 051003 (2015).

${ }^{14}$ F. Martinez-Pedrero, A. Ortiz-Ambriz, I. Pagonabarraga, and P. Tierno, Phys. Rev. Lett. 115, 138301 (2015).

${ }^{15}$ H. Massana-Cid, F. Martinez-Pedrero, E. Navarro-Argemí, I. Pagonabarraga, and P. Tierno, New J. Phys. 19, 103031 (2017).

${ }^{16}$ F. Peruani, A. Deutsch, and M. Bär, Phys. Rev. E 74, 030904 (2006).

${ }^{17}$ I. Buttinoni, J. Bialké, F. K. ümmel, H. Löowen, C. Bechinger, and T. Speck, Phys. Rev. Lett. 110, 238301 (2013).

${ }^{18}$ J. Stenhammar, D. Marenduzzo, R. J. Allen, and M. E. Cates, Soft Matter 10, 1489 (2014)
${ }^{19}$ A. Zöttl and H. Stark, Phys. Rev. Lett. 112, 118101 (2014).

${ }^{20}$ J. Elgeti, R. G. Winkler, and G. Gompper, Rep. Prog. Phys. 78, 056601 (2015).

${ }^{21}$ N. Yoshinaga and T. B. Liverpool, Phys. Rev. E 96, 020603 (2017).

${ }^{22}$ F. Ginot, I. Theurkauff, F. Detcheverry, C. Ybert, and C. Cottin-Bizonne, Nat. Commun. 9, 696 (2018).

${ }^{23}$ H. Massana-Cid, J. Codina, I. Pagonabarraga, and P. Tierno, Proc. Natl. Acad. Sci. U. S. A. 115, 10618 (2018)

${ }^{24}$ F. Martinez-Pedrero, E. Navarro-Argemí, A. Ortiz-Ambriz, I. Pagonabarraga, and P. Tierno, Sci. Adv. 4, eaap9379 (2018).

${ }^{25}$ S. H. Lee and C. M. Liddell, Small 5, 1957 (2009).

${ }^{26}$ J. C. Crocker and D. G. Grier, J. Colloid Interface Sci. 179, 298 (1996).

${ }^{27}$ F. Martinez-Pedrero, H. Massana-Cid, and P. Tierno, Small 13, 1603449 (2017).

${ }^{28}$ J. R. Blake and A. T. Chwang, J. Eng. Math. 8, 23 (1974).

${ }^{29}$ P. Tierno, Phys. Chem. Chem. Phys. 16, 23515 (2014).

${ }^{30}$ H. Morimoto, T. Ukai, Y. Nagaoka, N. Grobert, and T. Maekawa, Phys. Rev. E 78, 021403 (2008).

${ }^{31}$ C. E. S. L. Schmid, M. F. Schneider, T. Franke, and A. Alexander-Katz, Proc. Natl. Acad. Sci. U.S.A. 107, 535 (2010).

${ }^{32}$ T. O. Tasci, P. S. Herson, K. B. Neeves, and D. W. M. Marr, Nat. Commun. 7, 10225 (2016)

${ }^{33}$ F. J. Maier, T. Lachner, A. Vilfan, T. O. Tasci, K. B. Neeves, D. W. M. Marr, and T. M. Fischer, Soft matter 12, 9314 (2016).

${ }^{34}$ T. Yang, T. O. Tasci, K. B. Neeves, and D. W. M. Marr, Langmuir 33, 5932 (2017).

${ }^{35}$ M. Driscoll, B. Delmotte, M. Youssef, S. Sacanna, A. Donev, and P. Chaikin, Nat. Phys. 13, 375 (2017).

${ }^{36}$ F. Martinez-Pedrero, A. Cebers, and P. Tierno, Phys. Rev. Appl. 6, 034002 (2016).

${ }^{37}$ A. Bricard, J.-B. Caussin, N. Desreumaux, O. Dauchot, and D. Bartolo, Nature 503, 95 (2013).

${ }^{38}$ J. Yan, M. Han, J. Zhang, E. Luijten, and S. Granick, Nat. Mater. 15, 1095 (2016).

${ }^{39}$ F. Martinez-Pedrero, A. Cebers, and P. Tierno, Soft Matter 12, 3688 (2016). 